\title{
PROTEGIENDO AL PERSONAL DE LA SALUD EN LA PANDEMIA COVID-19
}

\author{
PROTECTING HEALTH PERSONNEL IN THE COVID-19 PANDEMIC
}

Jhony A. De La Cruz-Vargas 1,2,a

Publicado online el 21 de marzo de 2020

Published online March 21, 2020

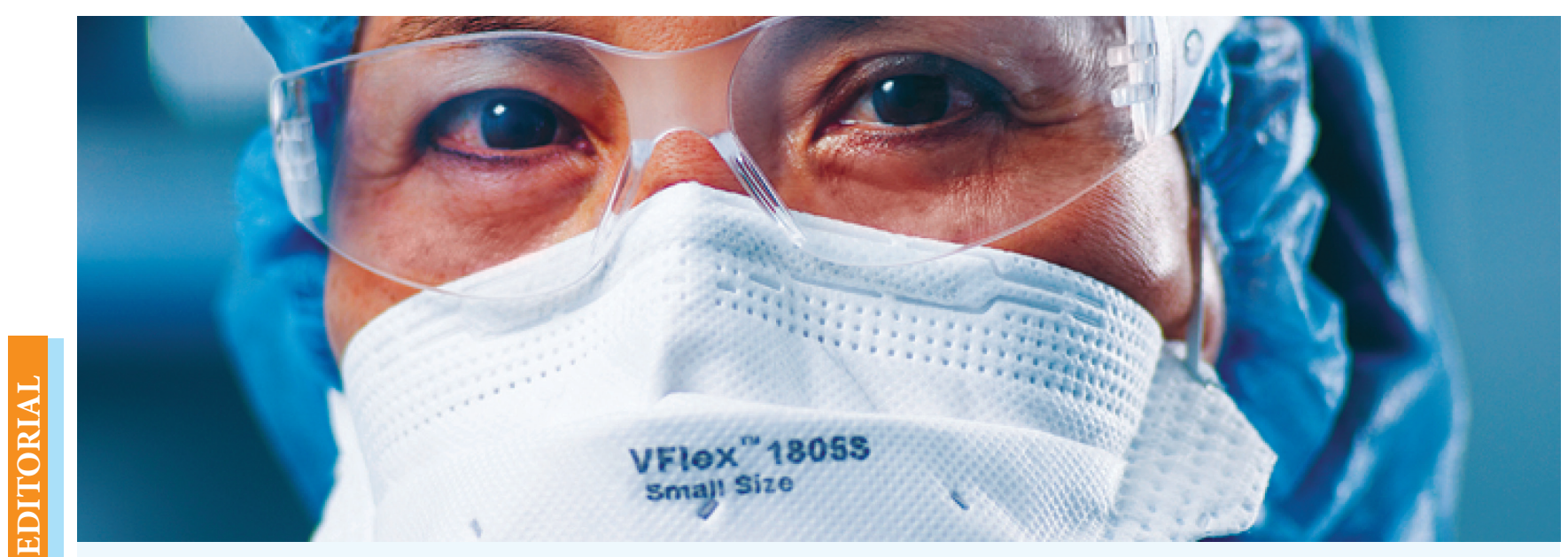

La epidemia y pandemia del COVID-19 constituyen una urgencia de salud pública sin precedentes, llegó a Latinoamérica, primero a Brasil, luego a Ecuador; los primeros casos en Perú fueron registrado a partir del 06 de marzo de 2020(1). La respuesta acertada del Gobierno del Perú decretó estado de emergencia nacional y aislamiento social obligatorio, inicialmente por 14 días, con el objetivo de reducir la exposición y trasmisión del coronavirus. Asignó fondos económicos para 3 millones de familias pobres en el Perú, durante el aislamiento social ${ }^{(2)}$.

Las repercusiones de COVID-19 son enormes, a nivel social, económico, cultural, educativo y por supuesto en el sistema de salud del país y su personal. Fronteras, universidades, comercios, restaurantes, cerrados. Las Administradoras de Fondos de Pensiones (AFP) anunciaron verse afectadas rápidamente, trasladando importantes pérdidas a los usuarios. Todo esto es impredecible y solo es la punta del iceberg.

Ahora mas que nuca, los médicos y los trabajadores de la salud son el recurso mas valioso de cada país.

El cuadro es claro, mientras millones de personas en todo el mundo se quedan en sus hogares, para minimizar la trasmisión del coronavirus y el síndrome respiratorio agudo severo, médicos y trabajadores de la salud se preparan para hacer exactamente lo contrario; ellos, irán a hospitales, clínicas y centros de salud, poniéndose en alto riesgo del COVID-19. Las experiencias en China e Italia nos muestran que el $20 \%$ de los trabajadores sanitarios se infectaron y solo en China, habían muerto 22 de ellos ${ }^{(3)}$.

La situación en los hospitales es compleja. No solo existe incertidumbre e intensa presión en los servicios de triaje, sino que aparece el agotamiento físico y mental, el tormento de las decisiones difíciles y el dolor de perder pacientes y colegas, todo esto además del riesgo de infección.

\footnotetext{
${ }^{1}$ Instituto de Investigación en Ciencias Biomédicas, Universidad Ricardo Palma, Lima -Perú.

${ }^{2}$ ISPOR-Perú.

a Doctor en Medicina.

Citar como: Jhony A. De La Cruz-Vargas. Protegiendo al personal de la salud en la pandemia COVID-19. Rev Rev. Fac. Med. Hum. Abril 2020; 20(2): 173-174. DOI 10.25176/RFMH.v20i2.2913 
Un punto esencial lo constituye la infraestructura y equipos disponibles en los hospitales y centros de salud. A medida que la pandemia se acelera, el acceso al equipo de protección personal (EPP) para los trabajadores de la salud es una preocupación clave. El personal médico tiene prioridad en muchos países, pero la escasez de EPP se ha descrito en las instalaciones mas afectadas $^{(4)}$.

Miembros del personal médico mientras esperan el equipo, atienden a que pueden estar infectados o reciben equipos que pueden no cumplir con los requisitos. Además de las preocupaciones por su seguridad personal, los trabajadores de la salud están ansiosos por el riesgo de trasmitir la infección a sus familias. Por si fuera poco, los trabajadores de salud que tengan en casa a padres ancianos o niños pequeños se verán afectados drásticamente por toda la situación.

Es probable que los sistemas de atención médica a nivel mundial podrían estar funcionando a una capacidad por arriba de la máxima durante muchos meses. Pero esta claro que los trabajadores de salud, a diferencia de los ventiladores o las salas, no pueden fabricarse con urgencia ni funcionan al $100 \%$ de su ocupación durante largos periodos.

Resulta esencial que los gobiernos vean a los médicos y trabajadores de la salud no simplemente como piezas de ajedrez, sino como personas humanas, con familias, con sueños y emociones. Dentro de la respuesta global, se debe garantizar la seguridad de los trabajadores de la salud. La provisión adecuada de EPP es solo el primer paso; se deben considerar otras medidas prácticas, incluida la cancelación de eventos no esenciales para priorizar los recursos, provisión de alimentos, descanso y apoyo familiar y psicológico. La tabla 1, nos muestra la realidad de casos de infección por COVID-19 en personal de salud al 20 de marzo de 2020.

Hoy nos toca cuidarlos a ellos.

Cuidemos a los que nos cuidaron en el pasado, nos cuidan en el presente y nos seguirán cuidando cuando la pandemia del coronovirus-19 haya pasado.

Tabla 1. Casos de infección por COVID-19 en personal de salud, Perú (al 20/03/2020 - 19:00 horas).

\begin{tabular}{|c|c|c|c|c|c|c|c|}
\hline Profesión/ocupación & EsSalud & Minsa & PNP & Privado & Otro & Total & $\%$ \\
\hline Médico residente & 4 & 3 & 1 & & & 8 & 33,33 \\
\hline Médico cirujano & 2 & 3 & & & 2 & 7 & 29,17 \\
\hline Enfermera & 1 & & & 1 & 1 & 3 & 12,50 \\
\hline Médico especialista & & 1 & & 1 & & 2 & 8,33 \\
\hline Obstreta & 1 & & & & & 1 & 4,17 \\
\hline Odontóloga & & & 1 & & & 1 & 4,17 \\
\hline Psicóloga & & & & & 1 & 1 & 4,17 \\
\hline Técnica de enfermería & 1 & & & & & 1 & 4,17 \\
\hline Total general & 9 & 7 & 2 & 2 & 4 & 24 & 100,00 \\
\hline
\end{tabular}

Nota: El 70,8\% de los casos de COVID-19 identificados en personal de salud, corresponden a médicos.

Fuente: Centro Nacional de Epidemiología, Prevención y Control de Enfermedades - MINSA.

Información sujeta a cambios de acuerdo a las investigaciones epidemiológicas que se realicen.

Correspondencia: Jhony A. De La Cruz Vargas.

Dirección: INICIB, Facultad de Medicina Humana, Pabellón J, Edificio Administrativo, 2do piso. Avenida Benavides 5440, Surco, Lima - Perú.

Teléfono: 708-0000/Anexo: 6016

Correo:jhony.delacruz@urp.edu.pe

\section{REFERENCIAS BIBLIOGRÁFICAS}

1. Coronavirus en el Perú. Plataforma digital única del estado peruano. https:// www.gob.pe/coronavirus

2. Rodriguez-Morales AJ, Gallego V, Escalera-Antezana JP, Mendez CA, Zambrano LI, Franco-Paredes C, Suárez JA, Rodriguez-Enciso HD, BalbinRamon GJ, Savio-Larriera E, Risquez A, Cimerman S, COVID-19 in Latin America: The implications of the first confirmed case in Brazil, Travel Medicine and Infectious Disease (2020), doi: https://doi.org/10.1016/j.tmaid.2020.101613.
3. The lancet. COVID-19: protecting health-care workers. Available online 19 March 2020. https://doi.org/10.1016/S0140-6736(20)30644-9

4. Bauchner $\mathrm{H}$, Fontanarosa PB, Livingston $\mathrm{EH}$. Conservación del suministro de equipo de protección personal: un llamado a ideas. JAMA Publicado en línea el 20 de marzo de 2020. http://jamanetwork.com/article.aspx?doi=10.1001/ jama.2020.4770 